\title{
Possible Flexoelectric Origin of the Lifshitz Transition in $\mathrm{LaAlO}_{3} / \mathrm{SrTiO}_{3}$ Interfaces
}

\author{
Amany Raslan and W. A. Atkinson* \\ Department of Physics \& Astronomy, Trent University, Peterborough Ontario, Canada, K9L 0G2
}

(Dated: August 14, 2018)

\begin{abstract}
Multiple experiments have observed a sharp transition in the band structure of $\mathrm{LaAlO}_{3} / \mathrm{SrTiO}_{3}$ (001) interfaces as a function of applied gate voltage. This Lifshitz transition, between a single occupied band at low electron density and multiple occupied bands at high density, is remarkable for its abruptness. In this work, we propose a mechanism by which such a transition might happen. We show via numerical modeling that the simultaneous coupling of the dielectric polarization to the interfacial strain ("electrostrictive coupling") and strain gradient ("flexoelectric coupling") generates a thin polarized layer whose direction reverses at a critical density. The Lifshitz transition occurs concomitantly with the polarization reversal and is first-order at $T=0$. A secondary Lifshitz transition, in which electrons spread out into semiclassical tails, occurs at a higher density.
\end{abstract}

$\mathrm{LaAlO}_{3}(\mathrm{LAO})$ and $\mathrm{SrTiO}_{3}(\mathrm{STO})$ are band insulators; however, when four or more monolayers of LAO are grown on top of a STO substrate, a mobile twodimensional electron gas (2DEG) forms on the STO side of the interface [1, 2]. One compelling feature of these interfaces is that the character of the 2DEG changes dramatically with the application of a gate voltage. Indeed, for (001) interfaces there is a narrow doping range over which the superconducting transition temperature [3-7], the spin-orbit coupling $7-10$, and the metamagnetic response [1] change by an order of magnitude. Furthermore, the superconducting gap and resistive transition appear at different temperatures at low electron densities, $n_{2 \mathrm{D}}$, but track each other closely at high $n_{2 \mathrm{D}} 12$. This qualitative distinction between low and high doping has also been seen in quantum dot transport experiments, which reveal a crossover from attractive to repulsive pairing interactions with increasing $n_{2 \mathrm{D}}[13$. While there is general agreement that the sensitivity to doping is connected to an observed Lifshitz transition [5, 14 17 between a single occupied band at low density and multiple occupied bands at high density [6, 17, 24, the mechanism by which this transition happens is not established.

Density functional theory (DFT), while instrumental in establishing fundamental interface properties $[25 \mid 28$, finds electron densities that are an order of magnitude larger than the Lifshitz transition density $n_{L} \sim 0.02-$ 0.05 electrons per 2D unit cell, and cannot easily be tuned through the transition. Schrödinger-Poisson calculations, for which $n_{2 \mathrm{D}}$ can be continuously tuned, persistently find multiple occupied bands even for $n_{2 \mathrm{D}} \ll n_{L}$ [5, 15, 29 31. Indeed, we showed previously that, because of STO is close to a ferroelectric (FE) quantum critical point 32, electrons become deconfined from an ideal interface as $n_{2 \mathrm{D}} \rightarrow 0$, and form a dilute quasi-threedimensional (quasi-3D) gas extending far into the STO substrate [33. This result is incompatible with experiments and raises the question, why is only a single band occupied at low $n_{2 \mathrm{D}}$ ?

Furthermore, the evolution of the band structure with $n_{2 \mathrm{D}}$ is highly unusual. Early work 14 showed that the filling of the lowest band is constant for $n_{2 \mathrm{D}}>n_{L}$, and recent experiments have found geometries in which its filling decreases [15] 17]. In the latter case, the first band actually empties itself into higher energy bands with increasing chemical potential. This cannot be understood within rigid-band models.

Moving beyond rigid bands, intra-atomic Coulomb (Hubbard) interactions 6, 24, have been invoked as a possible explanation for the unusual band filling [14, 16]; however, as we show below, these are too weak to be relevant. We suggest that a purely electronic explanation for the Lifshitz transition is unlikely, and focus instead on STO's unique dielectric properties. In particular, STO's proximity to a FE transition allows for a large coupling between the dielectric polarization and lattice strains 34. This makes STO interfaces qualitatively different from conventional metallic interfaces, and as we show enables a novel switchable state involving the lattice polarization and the 2DEG.

Strains arising from direct application of pressure or from lattice misfits between a thin film and its substrate form the basis of "strain engineering". By such methods, one can modify the dielectric response 35, 36, and even stabilize ferroelectricity [35, 37, in STO. Furthermore, strains applied to metallic LAO/STO interfaces can be used to tune their carrier density and subband occupations [38 40]. We distinguish between these extrinsic bulk strains, and the intrinsic strain that arises from $c$-axis relaxation (perpendicular to the interface) within a few $\mathrm{nm}$ of the LAO/STO interface. Because the LAO cap layer is grown on top of the STO substrate, the transverse (parallel to the interface) strain in the STO vanishes; however, there is a longitudinal strain $\eta(z) \sim 0.01-0.03$ extending four or five layers into the STO [41, 42]. This strain couples to the polarization $P$ through an electrostrictive contribution [35], $-g_{11} \eta P^{2}$, and a flexoelectric contribution [34], $-f_{11} P \partial_{z} \eta$, to the lattice free energy.

While flexoelectric effects are typically negligible in bulk materials, they appear to be a generic feature of nanometer structures in FEs-including domain walls 


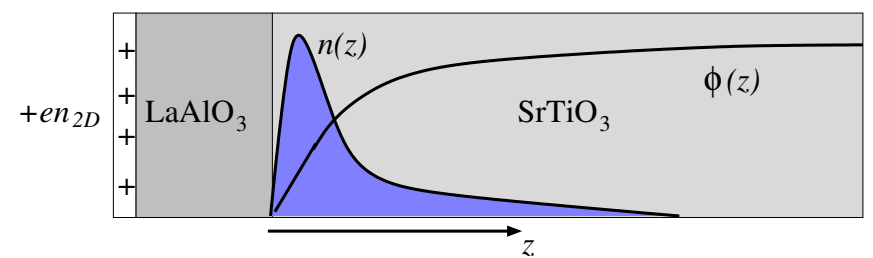

FIG. 1. Schematic LAO/STO interface showing the interface geometry, the electron potential energy $\phi(z)$ and electron density $n(z)$.

[43, 44], grain boundaries [45], and cracks [46]-where the strain gradient $\partial_{z} \eta$ is often enormous. Flexoelectricity substantially affects the performance of nanocapacitors [47, and numerous proposals for flexo-mechanical devices have been made [34. Whereas nearly all previous work has focused on insulating FEs, we show here that flexoelectricity fundamentally alters the 2DEG at STO interfaces.

We consider a model (001) interface between a STO substrate and a LAO cap layer [Fig. 1]. Several doping mechanisms, including electronic reconstruction [48, a nonstoichiometric LAO surface 49, 50, and top-gating, contribute to the 2DEG in the STO substrate. For our purposes, these doping mechanisms can be modeled by a positive charge density $e n_{2 \mathrm{D}}$ on the LAO surface, and overall neutrality requires a $2 \mathrm{D}$ charge density of $-e n_{2 \mathrm{D}}$ in the STO substrate. The 2DEG forms on the STO side of the interface owing to the wide LAO band gap [51, and is confined by a potential $\phi(z)$ that depends on $n_{2 \mathrm{D}}$ and on the dielectric screening in the substrate. Doped electrons reside on the $\mathrm{Ti} t_{2 g}$ orbitals.

The $t_{2 g}$ bands are obtained from a tight-binding Hamiltonian that has been fitted to Shubnikov-de Haas measurements in bulk STO [52, and the lattice polarization is obtained from a Landau-Devonshire free energy that has been fitted to bulk measurements of the dielectric function [30] (see the Supplemental Material [53]). The polarization and electronic eigenstates are coupled through the electric field, which is obtained from the Poisson equation. The model has a planar geometry, so that the polarization, electric field, etc., depend only on the distance $z$ from the interface; we discretize the model, so that there are $L$ layers of STO with, e.g. $P_{i}$ denoting the polarization in the $i$ th layer.

The Landau-Devonshire free energy is

$$
\mathcal{U}=\frac{1}{2} \sum_{i, j} P_{i} \tilde{D}_{i j} P_{j}-\sum_{i} \tilde{E}_{i} P_{i}+\text { quartic terms }
$$

where $i$ and $j$ are layer indices, and $\tilde{E}_{i}$ and $\tilde{D}_{i j}$ are linear and quadratic coefficients of the free energy expansion. The quartic terms in Eq. (1), which are parameterized by a coefficient $\gamma$ [53], are generally negligible in the doping range explored here [29, 30], but can be important when flexoelectric effects are included.
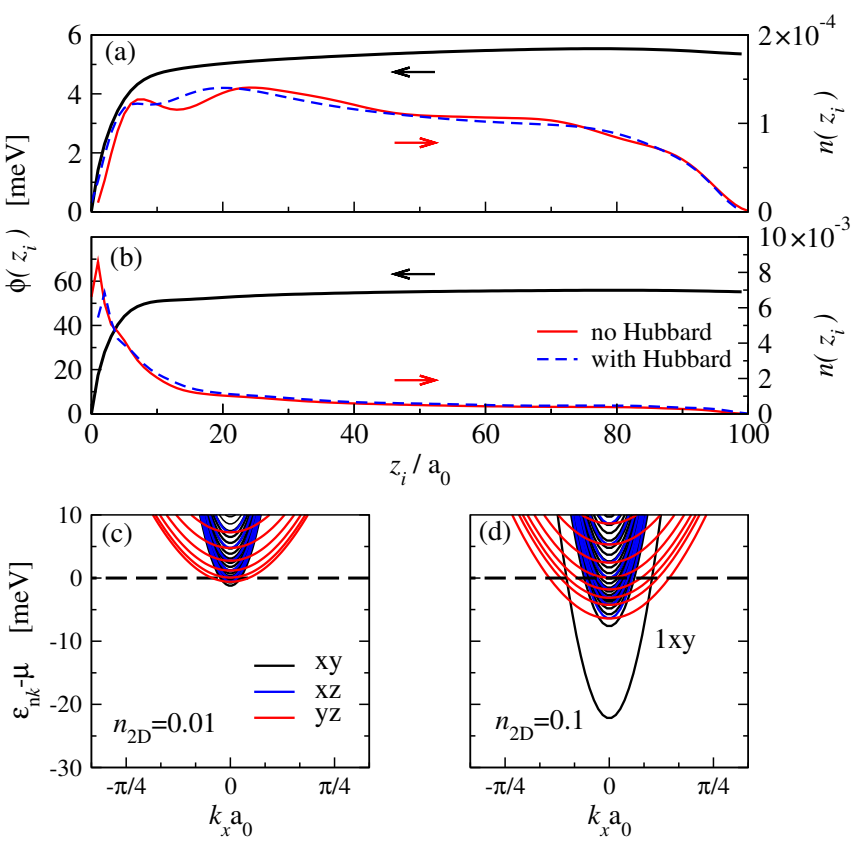

FIG. 2. The ideal interface. The confining potential and 3D charge density are plotted as functions of distance $z_{i}$ from the interface for (a) low $\left(n_{2 \mathrm{D}}=0.01\right)$ and (b) high $\left(n_{2 \mathrm{D}}=0.1\right)$ electron density. $\left[n_{2 \mathrm{D}}\right.$ and $n(z)$ are per $2 \mathrm{D}$ and $3 \mathrm{D}$ unit cell, respectively.] Curves labeled "with Hubbard" include intra-atomic interactions, with $U_{0}=4 \mathrm{eV}, U=2.4 \mathrm{eV}$, and $J=0.8 \mathrm{eV}$ [53; all other results have $U_{0}=U=J=0$. The corresponding band structures (without Hubbard interactions) are shown in (c) and (d). Unless otherwise stated, all results in this work are at $T=1 \mathrm{~K}$ and for $L=100$ layers.

Figure 2 illustrates the absence of a Lifshitz transition in an ideal interface. In this case, $\tilde{\mathbf{D}}$ is equal to the matrix $\mathbf{D}$ of stiffness constants for bulk STO, and $\tilde{E}_{i}=E_{i}$ is the total electric field in layer $i$. Figures 2(a) and 2(b) show the electron potential energy $\phi(z)$ and $3 \mathrm{D}$ density $n(z)$ for low and high values of $n_{2 \mathrm{D}}$. The key point is that the depth of the potential well confining the 2DEG is approximately proportional to $n_{2 \mathrm{D}}$, and at low density is too shallow to create $2 \mathrm{D}$ bound states. Thus, while most of the charge lies within $\sim 20$ unit cells $(8 \mathrm{~nm})$ of the interface when $n_{2 \mathrm{D}}=0.1$, it spreads far into the substrate when $n_{2 \mathrm{D}}=0.01$, with $n(z)$ decaying as a power law 33. (Densities are per 2D or 3D unit cell, as appropriate.) The electron confinement at high density is because a large fraction of the 2DEG occupies a single $2 \mathrm{D}$ band ("1xy"), while the deconfinement at low density is because the electrons are shared amongst a nearly continuous spectrum of subbands. That multiple bands are occupied at low $n_{2 \mathrm{D}}$ is inconsistent with experiments.

The situation is not substantively changed when Hubbard-like interactions are included, and indeed the charge distributions with and without Hubbard interactions are nearly the same [Fig. 2(a) and Fig. 2(b)].

The shortcomings of the ideal interface are corrected 
if strain is included. For a layer-dependent strain $\eta_{i}$,

$$
\tilde{D}_{i j}=D_{i j}-2 \delta_{i, j} g_{11} \eta_{i}, \quad \tilde{E}_{i}=E_{i}+\left.f_{11} \frac{\partial \eta}{\partial z}\right|_{z=z_{i}},
$$

with $g_{11}$ and $f_{11}$ the coupling constants, and $\delta_{i, j}$ the Kronecker delta-function. We adopt an empirical expression that qualitatively fits experimental measurements of the strain profile [41, 42, $\eta_{i}=\eta_{1} \exp \left[-\left(z_{i} / d\right)^{4}\right]$, with $\eta_{1} \sim 0.01-0.03$ the strain at the top STO layer and $d=4 a_{0}$. On general grounds, a piezoelectric term, $-e\left(z_{i}\right) \eta_{i}$ should also be added to $\tilde{E}_{i}$, where the coupling constant $e\left(z_{i}\right)$ vanishes away from the interface 43 . Because $e\left(z_{i}\right)$ is unknown, and because it plays the same qualitative role as flexoelectricity, surface piezoelectricity will not be considered explicitly.

Key to Eq. 22 is that, because $\partial_{z} \eta$ is negative, the effective field $E_{i}$ can be negative when the electric field $E_{i}$ is sufficiently small, provided $f_{11}>0$. In this case, the polarization at the interface will point oppositely to the external field, and towards the interface. This allows for a switchable polarization as a function of gate voltage.

Unless otherwise stated, we adopt the bulk value $g_{11}=$ $0.118 \epsilon_{0}^{-1}$, with $\epsilon_{0}$ the permittivity of free space [35, 53 . The appropriate value of $f_{11}$ is harder to asses [54 56], first because it is difficult to measure, even in bulk [56, second because surface corrections should be comparable to the bulk value 43, and third because screening by the 2DEG modifies the lattice response to a longitudinal strain gradient. $f_{11}$ is thus the only unknown parameter in our model. One empirical guide is a recent observation that the STO surface polarization in ungated samples points towards the interface [42, implying $f_{11}>0$. We then take $f_{11}$ of order a few $\mathrm{V}$ [34, which is typical for perovskites.

Figure 3 (a) shows the filling of the four lowest bands as a function of $n_{2 \mathrm{D}}$, along with the total filling of the remaining bands making up the tails of the charge distribution. There is a clear Lifshitz transition at $n_{L 1} \approx 0.0154$ at which the slope of $n_{1 x y}$ (the filling of the 1xy band) is discontinuous: all electrons reside in the 1xy band when $n_{2 \mathrm{D}}<n_{L 1}$, and $n_{1 x y}$ is nearly constant over an extended region when $n_{2 \mathrm{D}}>n_{L 1}$. That such a transition emerges naturally from the model without any parameter tuning, and that the predicted transition lies close to the experimental value of $n_{L}$, is remarkable.

There is a second transition at $n_{L 2} \approx 0.025$; this has a small effect on $n_{1 x y}$, and is primarily a redistribution of electrons from the $1 \mathrm{xz} / \mathrm{yz}$ and $2 \mathrm{xy}$ bands into the quasi$3 \mathrm{D}$ tails. Although no more than $20 \%$ of the charge occupies the tails, their 2D density of states is ordersof-magnitude larger than that of the 1xy band, which makes them a highly effective charge reservoir.

The transition at $n_{L 1}$ is sharp, suggesting that there are two competing ground states, and indeed the firstorder nature of the transition is illustrated by Fig. 3(b),
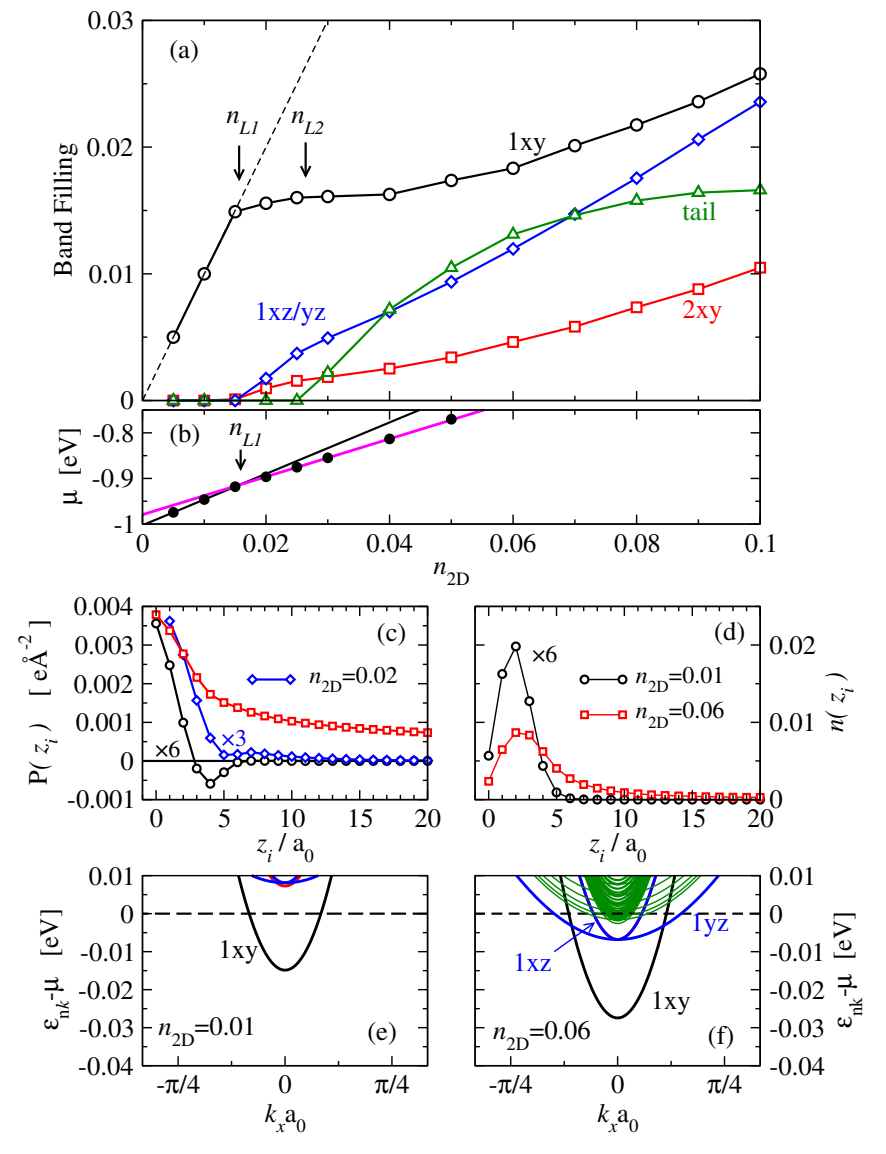

FIG. 3. Effects of strain on the 2DEG. (a) The fillings of the lowest four bands are shown individually, along with the cumulative filling of the remaining bands ("tail") and the total electron density (dashed line), as a function of $n_{2 \mathrm{D}}$. Bands are labeled $j \alpha$, with $j$ the band index and $\alpha$ the band symmetry. There are two Lifshitz transitions, at $n_{L 1}$ and $n_{L 2}$. (b) The chemical potential is plotted versus $n_{2 \mathrm{D}}$ (symbols). Black and magenta lines are linear fits to the regions $n_{2 \mathrm{D}}<n_{L 1}$ and $n_{L 1}<n_{2 \mathrm{D}}<n_{L 2}$, highlighting the discontinuity in slope at $n_{L 1}$. Any discontinuity at $n_{L 2}$ is too weak to see. The (c) polarization and (d) electron density are plotted for the top 20 STO layers for $n_{2 \mathrm{D}}$ below and above the transitions. A positive polarization points away from the interface. The corresponding band structures are shown in (e) and (f). In (f), the 2xy band is obscured by the 1yz band. The tail bands in (a) correspond to the dense spectrum of unlabeled bands in (f). Here, $f_{11}=2 \mathrm{~V}$ and $\eta_{1}=0.02$.

which shows a discontinuity in the inverse isothermal compressibility $\kappa_{T}^{-1} \propto d \mu / d n_{2 \mathrm{D}}$. There is, presumably, a second discontinuity at $n_{L 2}$; however it is too small to resolve in our data.

To characterize the states on either side of the transition, we show in Fig. 3(c) and Fig. 3(d) the lattice polarization and electron distribution, respectively. The main feature of this figure is that for $n_{2 \mathrm{D}}<n_{L 1}$, there is a thin layer of negative polarization that extends over the region $3 a_{0} \leq z_{i} \leq 6 a_{0}$, where the strain gradient is largest. In this region, the polarization points towards the inter- 


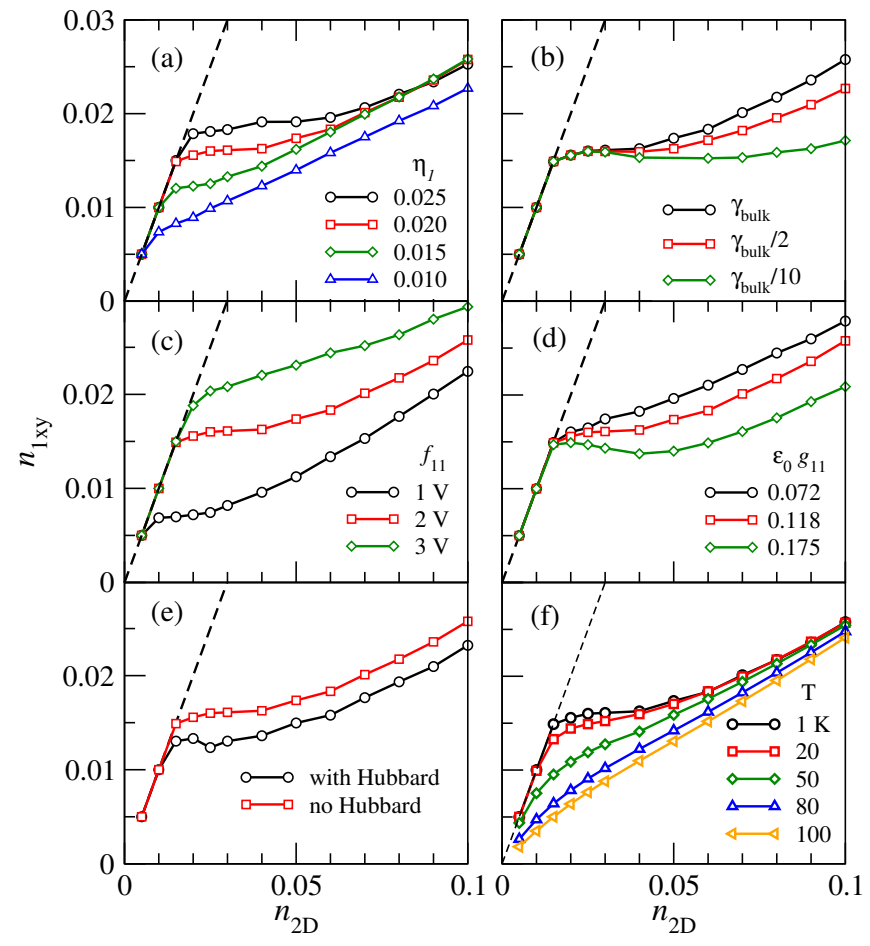

FIG. 4. Factors influencing the band filling. The 1xy band filling is shown for different values of (a) the interfacial strain, (b) the coefficient of the quartic term in Eq. (1), (c) the flexoelectric constant, and (d) the electrostrictive constant. In (e), cases with and without Hubbard-like interactions are compared. The "with Hubbard" calculations assume $U_{0}=4 \mathrm{eV}$, $U=2.4 \mathrm{eV}$, and $J=0.8 \mathrm{eV}$. In (f), $n_{1 x y}$ is shown for different temperatures. Default model parameters are as in Fig. 3 and $\gamma_{\text {bulk }}=2750 \mathrm{eV}^{5} e^{-4}$ is the estimate for $\gamma$ in bulk samples reported in 30].

face, and opposite to the electric field. This enhances the confining potential, and pulls electrons into a narrow 2D layer sandwiched between the interface and the region of negative polarization [Fig. 3(c)]. When $n_{2 \mathrm{D}}>n_{L 1}$, the electric field is strong enough to overcome flexoelectric effects, and the polarization switches abruptly to be positive everywhere. In this regime, the electrons move away from the interface and begin to occupy other bands. When $n_{2 \mathrm{D}}>n_{L 2}$, the tail states become occupied, and the polarization develops a power-law decay into the substrate [33. The corresponding band structures show a transition from a single occupied $d_{x y}$ band at low density, to multiple occupied bands at high density [Figs. 3. (e) and [3(f)].

Figure 4 shows different factors affecting $n_{1 x y}$. Both the interfacial strain [Fig. 4(a)] and the flexoelectric coupling constant [Fig. 4(c)] have a strong impact on the critical doping, consistent with our earlier assertion that the polarization switches direction at the value of $n_{L 1}$ where the electric field exceeds the flexoelectric term in Eq. (2). That $n_{L 1}$ depends on strain provides a natural explanation for the observed variability between samples of the critical doping.

Conversely, neither the quartic coefficient $\gamma$ [Fig. 4(b)] nor the electrostrictive coupling constant $g_{11}$ [Fig. 4 4 (d)] has a significant effect on $n_{L 1}$. Rather, they determine the behavior of $n_{1 x y}$ on the high-density side of the transition. The quartic term, which progressively reduces the dielectric screening as $n_{2 \mathrm{D}}$ grows, is responsible for an upturn in $n_{1 x y}$ at large $n_{2 \mathrm{D}}$, while $g_{11}$ principally affects the slope on the high density side of the Lifshitz transition, with large values of $g_{11}$ actually leading to a decline in $n_{1 x y}$ with increasing $n_{2 \mathrm{D}}$.

The slope of $n_{1 x y}$ above the Lifshitz transition is also strongly affected by the doping-dependence of the interfacial strain. In Fig. 4(a), the strain is fixed for each curve; however, the strain is not constant in real interfaces and, for example, shrinks with increasing LAO thickness [42]. The effects of gating have not been reported; however, one may infer from Fig. 4(a) that if the strain were to relax with increasing $n_{2 \mathrm{D}}$, the slope above the transition would be negative, as found in some experiments [15-17.

Figure 4(e) shows that the inclusion of intra-atomic interactions has a small quantitative effect on $n_{1 x y}$, but does not change qualitative features of the transition. This is consistent with our general finding that electron densities are too small for Hubbard-like interactions to have a significant effect.

Figure $4(\mathrm{f})$ shows the evolution of $n_{1 x y}$ with $T$. While temperature appears directly in the calculation of the band fillings, the most important effect is in the stiffness coefficients $D_{i j}$, which reflect STO's strongly $T$ dependent dielectric function 53 . In Fig. $4(\mathrm{f})$, the transition changes very little for $T \lesssim 20 \mathrm{~K}$, over which range $D_{i j}$ is nearly constant, and then is gradually wiped out as the temperature is further raised. The mechanism for the wipeout is straightforward; as $T$ increases, the lattice stiffens (i.e. the permittivity decreases), and the spontaneously polarized layer near the interface disappears.

The predictions made here can be directly tested by atomic-resolution probes that can resolve the interface polarization. Notably, Lee et al. [42] observed a "headto-head" arrangement of LAO and STO polarizations in ungated samples, which they associated with the interfacial doping mechanism. In their measurements, the reversed polarization on the STO side of the interface extends $\sim 5$ unit cells into the substrate, similar to what we find. In this regard, it would be extremely interesting to see whether the measured polarization can be switched by an external gate voltage, and to determine whether this correlates with a Lifshitz transition.

In summary, we have argued that LAO/STO interfaces can be thought of as a metallic system with ferroelectric characteristics arising from interfacial strains. This is reminiscent of an earlier proposal that the 2DEG at interfaces beween $\mathrm{LAO}$ and a bulk ferroelectric, $\mathrm{BaTiO}_{3}$, should exhibit a switchable metallic state [57]; in that case, the external electric field due to the LAO cap layer 
was ultimately shown suppress switchability [58. The current model differs in two key respects: here, (i) switchability is a consequence of the competition between flexoelectric effects and the external field, and (ii) the switchable region extends over only a few unit cells.

We thank A. E. M. Smink for helpful comments. We acknowledge support by the Natural Sciences and Engineering Research Council (NSERC) of Canada. A. R. was supported by an Ontario Graduate Scholarship.

\section{* billatkinson@trentu.ca}

[1] A. Ohtomo and H. Y. Hwang, "A high-mobility electron gas at the $\mathrm{LaAlO}_{3} / \mathrm{SrTiO}_{3}$ heterointerface," Nature Commun. 427, 423-426 (2004).

[2] S. Thiel, G. Hammerl, A. Schmehl, C. W. Schneider, and J. Mannhart, "Tunable Quasi-Two-Dimensional Electron Gases in Oxide Heterostructures," Science 313, 1942-1945 (2006).

[3] A. D. Caviglia, S. Gariglio, N. Reyren, D. Jaccard, T. Schneider, M. Gabay, S. Thiel, G. Hammerl, J. Triscone, and J. M. Mannhart, "Electric field control of the $\mathrm{LaAlO}_{3} / \mathrm{SrTiO}_{3}$ interface ground state," Nature 456, 624-627 (2008).

[4] D. A. Dikin, M. Mehta, C. W. Bark, C. M. Folkman, C. B. Eom, and V. Chandrasekhar, "Coexistence of Superconductivity and Ferromagnetism in Two Dimensions," Phys. Rev. Lett. 107, 056802 (2011).

[5] J. Biscaras, N. Bergeal, S. Hurand, C. Grossetete, A. Rastogi, R. C. Budhani, D. LeBoeuf, C. Proust, and J. Lesueur, "Two-Dimensional Superconducting Phase in $\mathrm{LaTiO}_{3} / \mathrm{SrTiO}_{3}$ Heterostructures Induced by HighMobility Carrier Doping," Phys. Rev. Lett. 108 (2012).

[6] E. Maniv, M. Ben Shalom, A. Ron, M. Mograbi, A. Palevski, M. Goldstein, and Y. Dagan, "Strong correlations elucidate the electronic structure and phase diagram of $\mathrm{LaAlO}_{3} / \mathrm{SrTiO}_{3}$ interface," Nature Commun. 6, 8239 (2015).

[7] S. Hurand, A. Jouan, C. Feuillet-Palma, G. Singh, J. Biscaras, E. Lesne, N. Reyren, A. Barthélémy, M. Bibes, J. E. Villegas, C. Ulysse, X. Lafosse, M. Pannetier-Lecoeur, S. Caprara, M. Grilli, J. Lesueur, and N. Bergeal, "Fieldeffect control of superconductivity and Rashba spin-orbit coupling in top-gated $\mathrm{LaAlO}_{3} / \mathrm{SrTiO}_{3}$ devices," Sci. Rep. 5, 12759 (2015).

[8] A. D. Caviglia, M. Gabay, S. Gariglio, N. Reyren, C. Cancellieri, and J. M. Triscone, "Tunable Rashba Spin-Orbit Interaction at Oxide Interfaces," Phys. Rev. Lett. 104, 126803 (2010).

[9] M. Ben Shalom, M. Sachs, D. Rakhmilevitch, A. Palevski, and Y. Dagan, "Tuning Spin-Orbit Coupling and Superconductivity at the $\mathrm{SrTiO}_{3} / \mathrm{LaAlO}_{3}$ Interface: A Magnetotransport Study," Phys. Rev. Lett. 104, 126802 (2010).

[10] Haixing Liang, Long Cheng, Laiming Wei, Zhenlin Luo, Guolin Yu, Changgan Zeng, and Zhenyu Zhang, "Nonmonotonically tunable Rashba spin-orbit coupling by multiple-band filling control in $\mathrm{SrTiO}_{3}$-based interfacial delectron gases," Phys. Rev. B 92, 3414 (2015).

[11] Arjun Joshua, Jonathan Ruhman, Sharon Pecker, Ehud Altman, and Shahal Ilani, "Gate-tunable polarized phase of two-dimensional electrons at the $\mathrm{LaAlO}_{3} / \mathrm{SrTiO}_{3}$ interface," Proc. Nat. Acad. Sci. 110, 9633-9638 (2013).

[12] C. Richter, H. Boschker, W. Dietsche, E. Fillis-Tsirakis, R. Jany, F. Loder, L. F. Kourkoutis, D. A. Muller, J. R. Kirtley, C. W. Schneider, and J. Mannhart, "Interface superconductor with gap behaviour like a high-temperature superconductor," Nature 502, 528-531 (2013).

[13] Guanglei Cheng, Michelle Tomczyk, Alexandre B Tacla, Hyungwoo Lee, Shicheng Lu, Josh .P Veazey, Mengchen Huang, Patrick Irvin, Sangwoo Ryu, Chang-Beom Eom, Andrew Daley, David Pekker, and Jeremy Levy, "Tunable Electron-Electron Interactions in $\mathrm{LaAlO}_{3} / \mathrm{SrTiO}_{3}$ Nanostructures," Phys. Rev. X 6, 041042 (2016).

[14] Arjun Joshua, S. Pecker, J. Ruhman, E. Altman, and S. Ilani, "A universal critical density underlying the physics of electrons at the $\mathrm{LaAlO}_{3} / \mathrm{SrTiO}_{3}$ interface," Nature Commun. 3, 1129 (2012).

[15] A. E. M. Smink, J. C. de Boer, M. P. Stehno, A. Brinkman, W. G. van der Wiel, and H. Hilgenkamp, "Gate-tunable band structure of the $\mathrm{LaAlO}_{3} / \mathrm{SrTiO}_{3}$ interface," Phys. Rev. Lett. 118, 106401 (2017).

[16] Wei Niu, Yu Zhang, Yulin Gan, Dennis V. Christensen, Merlin V. Soosten, Eduardo J. Garcia-Suarez, Anders Riisager, Xuefeng Wang, Yongbing Xu, Rong Zhang, Nini Pryds, and Yunzhong Chen, "Giant tunability of the two-dimensional electron gas at the interface of $\gamma$ $\mathrm{Al}_{2} \mathrm{O}_{3} / \mathrm{SrTiO}_{3}$," Nano Lett. 17, 6878-6885 (2017).

[17] A. E. M. Smink, M. P. Stehno, J. C. de Boer, A. Brinkman, W. G. van der Wiel, and H. Hilgenkamp, "Correlation between superconductivity, band filling and electron confinement at the $\mathrm{LaAlO}_{3}-\mathrm{SrTiO}_{3}$ interface," Phys. Rev. B 97, 245113 (2018).

[18] Younghyun Kim, Roman M Lutchyn, and Chetan Nayak, "Origin and transport signatures of spin-orbit Interactions in one- and two-dimensional $\mathrm{SrTiO}_{3}$-based heterostructures," Phys. Rev. B 87, 245121 (2013).

[19] Zhicheng Zhong, Anna Tóth, and Karsten Held, "Theory of spin-orbit coupling at $\mathrm{LaAlO}_{3} / \mathrm{SrTiO}_{3}$ interfaces and $\mathrm{SrTiO}_{3}$ surfaces," Phys. Rev. B 87, 161102 (2013).

[20] Guru Khalsa, Byounghak Lee, and A. H. MacDonald, "Theory of $t_{2 g}$ electron-gas Rashba interactions," Phys. Rev. B 88, 041302 (2013).

[21] Mark H. Fischer, Srinivas Raghu, and Eun-Ah Kim, "Spin-orbit coupling in $\mathrm{LaAlO}_{3} / \mathrm{SrTiO}_{3}$ interfaces: magnetism and orbital ordering," New J. Phys. 15, 023022 (2013).

[22] Jianhui Zhou, Wen-Yu Shan, and Di Xiao, "Spin responses and effective Hamiltonian for the two dimensional electron gas at oxide interface $\mathrm{LaAlO}_{3} / \mathrm{SrTiO}_{3}$," Phys. Rev. B 91, 241302 (2015).

[23] Yasuharu Nakamura and Youichi Yanase, "MultiOrbital Superconductivity in $\mathrm{SrTiO}_{3} / \mathrm{LaAlO}_{3}$ Interface and $\mathrm{SrTiO}_{3}$ Surface," J. Phys. Soc. Jpn. 82, 083705 (2013).

[24] S. Nandy, N. Mohanta, S. Acharya, and A. Taraphder, "Anomalous transport near the Lifshitz transition at the $\mathrm{LaAlO}_{3} / \mathrm{SrTiO}_{3}$ interface," Phys. Rev. B 94, 155103 (2016).

[25] Zoran Popović, Sashi Satpathy, and Richard Martin, "Origin of the two-dimensional electron gas carrier density at the $\mathrm{LaAlO}_{3}$ on $\mathrm{SrTiO}_{3}$ interface," Phys. Rev. Lett. 101, 256801 (2008).

[26] Won-Joon Son, Eunae Cho, Bora Lee, Jaichan Lee, and Seungwu Han, "Density and spatial distribution of charge 
carriers in the intrinsic $n$-type $\mathrm{LaAlO}_{3}-\mathrm{SrTiO}_{3}$ interface," Phys. Rev. B 79, 245411 (2009).

[27] Rossitza Pentcheva and Warren E. Pickett, "Avoiding the polarization catastrophe in $\mathrm{LaAlO}_{3}$ overlayers on $\mathrm{SrTiO}_{3}(001)$ through polar distortion," Phys. Rev. Lett. 102, 107602 (2009).

[28] Massimiliano Stengel, "First-principles modeling of electrostatically doped perovskite systems," Phys. Rev. Lett. 106, 136803 (2011).

[29] Guru Khalsa and A. H. MacDonald, "Theory of the $\mathrm{SrTiO}_{3}$ surface state two-dimensional electron gas," Phys. Rev. B 86, 125121 (2012).

[30] Amany Raslan, Patrick Lafleur, and W. A. Atkinson, "Temperature-dependent band structure of $\mathrm{SrTiO}_{3}$ interfaces," Phys. Rev. B 95, 054106 (2017).

[31] Danfeng Li, Sébastien Lemal, Stefano Gariglio, Zhenping Wu, Alexandre Fête, Margherita Boselli, Philippe Ghosez, and Jean-Marc Triscone, "Probing Quantum Confinement and Electronic Structure at Polar Oxide Interfaces," Advanced Science 4, 1800242 (2018).

[32] S. E. Rowley, L. J. Spalek, R. P. Smith, M. P. M. Dean, M. Itoh, J. F. Scott, G. G. Lonzarich, and S. S. Saxena, "Ferroelectric quantum criticality," Nature Phys. 10, 367372 (2014).

[33] W. A. Atkinson, Patrick Lafleur, and Amany Raslan, "Influence of the ferroelectric quantum critical point on $\mathrm{SrTiO}_{3}$ interfaces," Phys. Rev. B 95, 054107 (2017).

[34] Pavlo Zubko, Gustau Catalan, and Alexander K Tagantsev, "Flexoelectric Effect in Solids," Ann. Rev. Mat. Res. 43, 387-421 (2013).

[35] Hiromoto Uwe and Tunetaro Sakudo, "Stress-induced ferroelectricity and soft phonon modes in $\mathrm{SrTiO}_{3}, "$ Phys. Rev. B 13, 271-286 (1976).

[36] Ruiping Wang, Norihiko Sakamoto, and Mitsuru Itoh, "Effects of pressure on the dielectric properties of $\mathrm{SrTi}^{18} \mathrm{O}_{3}$ and $\mathrm{SrTi}^{16} \mathrm{O}_{3}$ single crystals ," Phys. Rev. B 62, 3577-3580 (2000).

[37] J. H. Haeni, P. Irvin, W. Chang, R. Uecker, P. Reiche, Y. L. Li, S. Choudhury, W. Tian, M. E. Hawley, B. Craigo, A. K. Tagantsev, X. Q. Pan, S. K. Streiffer, L. Q. Chen, S. W. Kirchoefer, J. Levy, and D. G. Schlom, "Roomtemperature ferroelectricity in strained $\mathrm{SrTiO}_{3}$," Nature Commun. 430, 758-761 (2004).

[38] C. W. Bark, D. A. Felker, Yuxuan Wang, Y. Zhang, H. W. Jang, C. M. Folkman, J. W. Park, S. H. Baek, H. Zhou, D. D. Fong, X. Q. Pan, E. Y. Tsymbal, M. S. Rzchowski, and C. B. Eom, "Tailoring a two-dimensional electron gas at the $\mathrm{LaAlO}_{3} / \mathrm{SrTiO}_{3}$ (001) interface by epitaxial strain," Proc. Nat. Acad. Sci. 108, 4720-4724 (2011).

[39] Maziar Behtash, Safdar Nazir, Yaqin Wang, and Kesong Yang, "Polarization effects on the interfacial conductivity in $\mathrm{LaAlO}_{3} / \mathrm{SrTiO}_{3}$ heterostructures: a first-principles study," Phys. Chem. Chem. Phys. 18, 6831-6838 (2016).

[40] Patrick Seiler, Jone Zabaleta, Robin Wanke, Jochen Mannhart, Thilo Kopp, and Daniel Braak, "Antilocalization at an oxide interface," Phys. Rev. B 97, 199 (2018).

[41] P. R. Willmott, S. A. Pauli, R. Herger, C. M. Schlepütz, D. Martoccia, B. D. Patterson, B. Delley, R. Clarke, D. Kumah, C. Cionca, and Y. Yacoby, "Structural basis for the conducting interface between $\mathrm{LaAlO}_{3}$ and $\mathrm{SrTiO}_{3}$," Phys. Rev. Lett. 99, 155502 (2007).

[42] P. W. Lee, V. N. Singh, H. J. Liu, J. C. Lin, C. H. Chen, G. Y. Guo, Y. H. Chu, and M. W. Chu, "Hidden lattice instabilities as origin of the conductive interface between insulating LaAlO," Nature Commun. 7, 12773 (2016).

[43] P. V. Yudin and A. K. Tagantsev, "Fundamentals of flexoelectricity in solids," Nanotechnology 24, 432001 (2013).

[44] Yijia Gu, Menglei Li, Anna N. Morozovska, Yi Wang, Eugene A. Eliseev, Venkatraman Gopalan, and LongQing Chen, "Flexoelectricity and ferroelectric domain wall structures: Phase-field modeling and DFT calculations," Phys. Rev. B 89, 2069 (2014).

[45] Peng Gao, Shuzhen Yang, Ryo Ishikawa, Ning Li, Bin Feng, Akihito Kumamoto, Naoya Shibata, Pu Yu, and Yuichi Ikuhara, "Atomic-Scale Measurement of Flexoelectric Polarization at $\mathrm{SrTiO}_{3}$ Dislocations," Phys. Rev. Lett. 120, 267601 (2018).

[46] Amir Abdollahi, Christian Peco, Daniel Millán, Marino Arroyo, Gustau Catalan, and Irene Arias, "Fracture toughening and toughness asymmetry induced by flexoelectricity," Phys. Rev. B 92, 2069 (2015).

[47] M. S. Majdoub, R. Maranganti, and P. Sharma, "Understanding the origins of the intrinsic dead layer effect in nanocapacitors," Phys. Rev. B 79, 502 (2009).

[48] Naoyuki Nakagawa, Harold Y. Hwang, and David A. Muller, "Why some interfaces cannot be sharp," Nature Mat. 5, 204-209 (2006).

[49] N. C. Bristowe, Philippe Ghosez, P. B. Littlewood, and Emilio Artacho, "The origin of two-dimensional electron gases at oxide interfaces: insights from theory," J. Phys. Cond. Mat. 26, 143201 (2014).

[50] I. I. Piyanzina, V. Eyert, Yu V. Lysogorskiy, D. A. Tayurskii, and T. Kopp, "Oxygen vacancies and hydrogen doping in $\mathrm{LaAlO}_{3} / \mathrm{SrTiO}_{3}$ heterostructures: electronic properties and impact on surface and interface reconstruction," (2018), arXiv:1803.01382

[51] S. Gariglio, A. Fête, and J. M. Triscone, "Electron confinement at the $\mathrm{LaAlO}_{3} / \mathrm{SrTiO}_{3}$ interface," J. Phys. Cond. Mat. 27, 283201 (2015).

[52] S. James Allen, Bharat Jalan, SungBin Lee, Daniel G. Ouellette, Guru Khalsa, Jan Jaroszynski, Susanne Stemmer, and Allan H. MacDonald, "Conduction-band edge and Shubnikov-de Haas effect in low-electron-density $\mathrm{SrTiO}_{3}, "$ Phys. Rev. B 88, 045114 (2013).

[53] See supplemental material for a detailed description of model.

[54] Jiawang Hong and David Vanderbilt, "First-principles theory of frozen-ion flexoelectricity," Phys. Rev. B 84, 2069 (2011).

[55] JiawProc. Nat.ang Hong, G. Catalan, J. F. Scott, and E. Artacho, "The flexoelectricity of barium and strontium titanates from first principles," J. Phys. Cond. Mat. 22, 112201 (2010).

[56] P. Zubko, G. Catalan, A. Buckley, P. R. L. Welche, and J. F. Scott, "Strain-gradient-induced polarization in $\mathrm{SrTiO}_{3}$ single crystals," Phys. Rev. Lett. 99, 520 (2007); "Erratum: Strain-gradient-induced polarization in $\mathrm{SrTiO}_{3}$ single crystals [phys. rev. lett. 99, 167601 (2007)]," 100, 199906 (2008).

[57] Manish K. Niranjan, Yong Wang, Sitaram S. Jaswal, and Evgeny Y. Tsymbal, "Prediction of a Switchable TwoDimensional Electron Gas at Ferroelectric Oxide Interfaces," Phys. Rev. Lett. 103, 1061 (2009).

[58] Yuxuan Wang, M. K. Niranjan, K. Janicka, J. P. Velev, M. Ye Zhuravlev, S. S. Jaswal, and E. Y. Tsymbal, "Ferroelectric dead layer driven by a polar interface," Phys. Rev. B 82, 1040 (2010). 


\section{SUPPLEMENTAL MATERIAL FOR: POSSIBLE FLEXOELECTRIC ORIGIN OF THE LIFSHITZ TRANSITION IN LAALO ${ }_{3}$ SRTIO $_{3}$ INTERFACES}

\section{Electronic Hamiltonian}

The electronic Hamiltonian is

$$
\hat{H}=\hat{H}_{0}+\hat{H}_{V}+\hat{H}_{U} .
$$

In this expression, $\hat{H}_{0}$ is the kinetic energy portion of the Hamiltonian, $\hat{H}_{V}$ describes the potential energy due to the long-range Coulomb interaction, obtained by solving the Poisson equation, while $\hat{H}_{U}$ contains short-range (intra-atomic) Coulomb contributions of the Hubbard type.

We adopt a layered geometry consistent with the structure of STO interfaces; we assume a (001) interface, such that there is translational invariance in the $x-y$ plane, and quantities such as the charge density, lattice polarization, and electrostatic potential depend only on the distance $z$ from the interface. For this geometry,

$$
\hat{H}_{0}=\sum_{i j \mathbf{k}} \sum_{\alpha \sigma} c_{\alpha \mathbf{k} i \sigma}^{\dagger} t_{i j}^{\alpha}(\mathbf{k}) c_{\alpha \mathbf{k} j \sigma}
$$

where $i$ and $j$ label $\mathrm{TiO}_{2}$ layers, and $\mathbf{k}=\left(k_{x}, k_{y}\right)$ is the $2 \mathrm{D}$ wavevector describing the motion of electrons parallel to the interface. The operator $c_{\alpha \mathbf{k} j \sigma}$ annihilates an electron with orbital type $\alpha$, spin $\sigma$, and wavevector $\mathbf{k}$ in layer $j$. The matrix element $t_{i j}^{\alpha}(\mathbf{k})$ is a hopping matrix element between orbitals of type $\alpha$.

There are two distinct hopping processes: hopping between neighboring orbitals of type $\alpha$ is large if the electron moves in the plane parallel to $\alpha$ (e.g. the $x-y$ plane for $d_{x y}$ orbitals), and small if it moves perpendicular to $\alpha$. The matrix elements are denoted by $t^{\|}$and $t^{\perp}$, respectively, and values are given in Table If We thus have

$$
\begin{aligned}
& t_{i j}^{x y}(\mathbf{k})=-2\left(t^{\|} c_{x}+t^{\|} c_{y}\right) \delta_{i, j}-t^{\perp} \delta_{\langle i, j\rangle}, \\
& t_{i j}^{x z}(\mathbf{k})=-2\left(t^{\|} c_{x}+t^{\perp} c_{y}\right) \delta_{i, j}-t^{\|} \delta_{\langle i, j\rangle}, \\
& t_{i j}^{y z}(\mathbf{k})=-2\left(t^{\perp} c_{x}+t^{\|} c_{y}\right) \delta_{i, j}-t^{\|} \delta_{\langle i, j\rangle},
\end{aligned}
$$

where $c_{n} \equiv \cos \left(k_{n} a_{0}\right)$ with $a_{0}$ the lattice constant, and $\delta_{\langle i, j\rangle}$ is one if $i$ and $j$ are nearest-neighbor planes, and zero otherwise.

\section{Coulomb Potential}

The long-range Coulomb term is

$$
\hat{H}_{V}=\sum_{i \alpha \sigma} \phi_{i} \hat{n}_{\alpha i \sigma}
$$

where $\hat{n}_{\alpha i \sigma}=N_{2 D}^{-1} \sum_{\mathbf{k}} c_{\alpha \mathbf{k} i \sigma}^{\dagger} c_{\alpha \mathbf{k} i \sigma}$ is the electron number operator for orbital type $\alpha$ and spin $\sigma$ in layer $i$, with

\begin{tabular}{l|r}
\hline \multicolumn{2}{c}{ Model parameters } \\
\hline$t^{\|}$ & $0.236 \mathrm{eV}$ \\
$t^{\perp}$ & $0.035 \mathrm{eV}$ \\
$a_{0}$ & $3.9 \AA$ \\
$D_{0}$ & $5.911 \times 10^{-3} \epsilon_{0}^{-1}$ \\
$D_{1}$ & $1.200 \times 10^{-3} \epsilon_{0}^{-1}$ \\
$D_{2}(T=0)$ & $0.312 \times 10^{-3} \epsilon_{0}^{-1}$ \\
$\alpha_{1}$ & $1.15 a_{0}$ \\
$\alpha_{2}$ & $5 a_{0}$ \\
$\gamma_{\text {bulk }}$ & $2750 \mathrm{eV} \AA^{5} \mathrm{e}^{-4}$ \\
$\epsilon_{\infty}$ & $5.5 \epsilon_{0}$ \\
$g_{11}$ & $0.118 \epsilon_{0}^{-1}$ \\
$f_{11}$ & $1-3 \mathrm{~V}$ \\
\hline
\end{tabular}

TABLE I. Model parameters used in our calculations. Hopping matrix elements $t^{\|}$and $t^{\perp}$ are from tight-binding fits [1] to Shubnikov-de Haas measurements in bulk STO 2]. Dielectric parameters $D_{0}, D_{1}, D_{2}, \alpha_{1}, \alpha_{2}$, and $\gamma_{\text {bulk }}$ are obtained from fits to the dielectric function. 3, 4, while $g_{11}$ is obtained from Ref. [5]. $\epsilon_{0}$ is the permittivity of free space and $-e$ is the electron charge.

$N_{2 D}$ the number of $2 \mathrm{D}$ unit cells. $\hat{H}_{V}$ contains a potential energy $\phi_{i} \equiv \phi\left(z_{i}\right)$ with contributions from three distinct interactions: electron-electron interactions between free carriers in the STO, electron-lattice interactions between free carriers and the STO polarization, and interactions between free carriers and the LAO surface charge.

The electrostatic potential energy (in SI units) is obtained by solving Poisson's equation,

$$
\epsilon_{\infty} \nabla \cdot \mathbf{E}(z)=\rho(z)-\nabla \cdot \mathbf{P}(z),
$$

where $\mathbf{E}(z), \mathbf{P}(z)$, and $\rho(z)$ are, respectively, the electric field, the lattice polarization, and the charge density, and $\epsilon_{\infty}$ is the optical dielectric constant. The charge density includes contributions from both the free carriers in the STO substrate and LAO surface charges. In the planar geometry, the polarization and electric field vectors are parallel to the $z$-axis, with $\mathbf{E}(z)=E(z) \hat{\mathbf{z}}, \mathbf{P}(z)=P(z) \hat{\mathbf{z}}$. Then, the potential energy is obtained from

$$
\phi(z)=\phi(0)+e \int_{0}^{z} d z^{\prime} E\left(z^{\prime}\right)
$$

Integrating Eq. (9) from a point outside the LAO surface (where $E$ and $P$ vanish) to layer $i$ inside the STO substrate yields

$$
\epsilon_{\infty} E_{i}=e n_{2 \mathrm{D}}-\frac{e}{a_{0}^{2}} \sum_{j<i} \sum_{\alpha \sigma} n_{\alpha j \sigma}-P_{i} .
$$

Equation 100 then gives the potential energy,

$$
\phi_{i}=\frac{e^{2}}{\epsilon_{\infty}}\left[n_{2 \mathrm{D}} z_{i}-\sum_{\substack{j<i \\ \alpha \sigma}} \frac{n_{\alpha j \sigma}}{a_{0}^{2}}\left(z_{i}-z_{j}\right)\right]-\frac{e a_{0}}{\epsilon_{\infty}} \sum_{j \leq i} P_{j},
$$


where $\phi(0)$ is set to zero.

We solve a discrete version of these equations, in which $E(z) \rightarrow E_{i}, P(z) \rightarrow P_{i}$, and

$$
\rho(z)=e n_{2 \mathrm{D}} \delta\left(z-z_{\mathrm{surf}}\right)-\frac{e}{a_{0}^{2}} \sum_{\alpha i \sigma} n_{\alpha i \sigma} \delta\left(z-z_{i}\right) .
$$

Here, $z_{\text {surf }}$ is the location of the LAO surface, $z_{i}=(i-$ 1) $a_{0}$ is the location of the $i$ th layer measured relative to the interface, and

$$
n_{\alpha i \sigma}=\left\langle\hat{n}_{\alpha i \sigma}\right\rangle .
$$

is the occupation of a single orbital $(\alpha, \sigma)$ in layer $i$.

The short-range Coulomb interaction on the Ti sites takes the form [6],

$$
\hat{H}_{U}=\sum_{\alpha \sigma i}\left[U_{0} n_{\alpha i \bar{\sigma}}+\sum_{\beta \neq \alpha, \sigma^{\prime}}\left(U-J \delta_{\sigma, \sigma^{\prime}}\right) n_{\beta i \sigma^{\prime}}\right] \hat{n}_{\alpha i \sigma},
$$

where $\bar{\sigma}=-\sigma$ and with the constraint

$$
U_{0}=U+2 J .
$$

In this expression, $U_{0}$ is the intra-orbital Hubbard interaction, and $U$ and $J$ are inter-orbital Hartree and exchange interactions. We constrain our calculations to eliminate the possibility of ferromagnetism, and require that $n_{\alpha i \downarrow}=n_{\alpha i \uparrow}$.

From the form of $\hat{H}_{U}$ [Eq. $[15$ ] ], it is clear that minimization of the intra-orbital Coulomb energy favors a spreading-out of charge between orbitals if $U_{0}$ and $J$ are large, and the collapse of charge into a single orbital type if $U$ is large. Indeed, we find that our calculations favor occupation of $d_{x y}$ orbitals over $d_{x z}$ and $d_{y z}$ orbitals when $J<U_{0} / 5$, and favor occupation of multiple orbital types when $J>U_{0} / 5$. The representative example shown in the manuscript takes $U_{0}=4 \mathrm{eV}$, and $J=0.8 \mathrm{eV}$ (so $J=U_{0} / 5$ ) and from the constraint (16), we obtain $U=2.4 \mathrm{eV}$. We have explored other $J$-values, and find that while details of the band structure change, our basic conclusions do not.

\section{Dielectric Model at Zero Temperature}

Dielectric screening comes from a soft optical phonon mode with a large dipole moment. The LandauDevonshire free energy for this mode is

$$
\mathcal{U}=\frac{1}{2} \sum_{i, j} P_{i} \tilde{D}_{i j} P_{j}-\sum_{i} \tilde{E}_{i} P_{i}+\text { quartic term }
$$

where $i$ and $j$ are layer indices, and $\tilde{E}_{i}$ and $\tilde{D}_{i j}$ are linear and quadratic coefficients of the free energy expansion. The quartic term is discussed below.
In bulk $\mathrm{STO}, \tilde{E}_{i}$ is equal to the electric field $E_{i}$; adjacent to the interface, inversion symmetry is broken and $\tilde{E}_{i}$ picks up additive corrections that extend over the first few STO layers. Similarly, the coefficients $\tilde{D}_{i j}$ equal their bulk values $D_{i j}$ away from the interface, but may be different near the interface.

Following our earlier work, we take the bulk coefficients

$$
D_{i j}= \begin{cases}D_{0}, & i=j \\ -D_{1} e^{-z_{i j}^{2} / 2 \alpha_{1}^{2}}-D_{2} e^{-z_{i j}^{2} / 2 \alpha_{2}^{2}}, & i \neq j\end{cases}
$$

where $z_{i j}=z_{i}-z_{j}$. Values for $D_{0}, D_{1}, D_{2}, \alpha_{1}$, and $\alpha_{2}$ that are valid at low $T$ are given in Table $\mathrm{I}$.

For a given electric field, the polarization $P_{\ell}$ is obtained by setting $\partial \mathcal{U} / \partial P_{\ell}=0$. In a bulk crystal with uniform electric field $E$ and polarization $P$, this yields $P=E / D_{\mathbf{q}=0}$ where $D_{\mathbf{q}=0}=\sum_{j} D_{i j}$. The bulk dielectric constant is then

$$
\epsilon=\epsilon_{\infty}+\frac{\partial P}{\partial E} \approx D_{\mathbf{q}=0}^{-1} .
$$

The final approximate equality arises because $\epsilon_{\infty}=5.5 \epsilon_{0}$ while $D_{\mathbf{q}=0}^{-1} \sim 10^{4} \epsilon_{0}$ at low $T$ ( $\epsilon_{0}$ is the permittivity of free space). In this dielectric model, the large value of $\epsilon$ comes from a near cancellation of the local $(i=j)$ and nonlocal $(i \neq j)$ contributions to $D_{\mathbf{q}=0}$.

Electrostrictive coupling through the term proportional to $g_{11}$ modifies the matrix $\tilde{\mathbf{D}}$ such that it has one or two negative eigenvalues, depending on the magnitude of the strain. In this instance, the quartic terms in Eq. (17) are required to stabilize the polarization. We find that the numerics are most easily controlled if we work in a basis in which $\tilde{\mathbf{D}}$ is diagonal. Letting $\Lambda_{n}$ and $\mathbf{S}$ be the eigenvalues and the matrix of eigenvectors of $\tilde{\mathbf{D}}$, we make the ansatz

$$
\mathcal{U}=\sum_{n}\left[\frac{1}{2} \Lambda_{n} \mathcal{P}_{n}^{2}-\mathcal{E}_{n} \mathcal{P}_{n}+\frac{\gamma}{4} \mathcal{P}_{n}^{4}\right]
$$

where $\mathcal{E}_{n}=\sum_{i} \tilde{E}_{i} S_{i n}$ and $\mathcal{P}_{n}=\sum_{i} P_{i} S_{i n}$. The first two terms in Eq. (20) are formally equivalent to Eq. (17), while the final term is an ansatz. The advantage of $\mathrm{Eq}(20)$ is that it is diagonal in the mode index $n$, and one can minimize each term in the sum analytically. Once $\mathcal{P}_{n}$ is known, then $P_{i}$ is obtained from $P_{i}=\sum_{n} S_{i n} \mathcal{P}_{n}$.

At low electron densities, $\mathcal{E}_{n}$ is sufficiently weak that the quartic term is negligible provided $\Lambda_{n}$ is positive and not too small; then, $\mathcal{P}_{n}=\mathcal{E}_{n} / \Lambda_{n}$, to a good approximation. For the one or two eigenmodes where $\Lambda_{n}$ is close to zero or negative, $\gamma$ cannot be neglected and the cubic equation obtained from setting

$$
\partial \mathcal{U} / \partial \mathcal{P}_{n}=\Lambda_{n} \mathcal{P}_{n}-\mathcal{E}_{n}+\gamma \mathcal{P}_{n}^{3}=0
$$

must be solved, with the solution that minimizes the energy of that eigenmode being selected. 


\section{Dielectric Model at Nonzero Temperature}

The temperature dependence of the dielectric function comes through the matrix elements $D_{i j}$, and our approach follows Raslan et al. [3]. The T-dependence is obtained in two steps. First, the measured dielectric susceptibility [4]. $\chi(T)$ is fitted to an empirical formula

$$
\chi_{\mathbf{q}=0}(T)=\left(\frac{T_{0}}{T_{s} \operatorname{coth}\left(T_{s} / T\right)}\right)^{\xi},
$$

where $T_{s}=15 \mathrm{~K}$ is the saturation temperature below which $\chi(T)$ becomes constant, and $T_{0}=1.46 \times 10^{4} \mathrm{~K}$ and $\xi=1.45$ are fitting parameters. Next, using

$$
\chi_{\mathbf{q}=0}(T)=\frac{1}{\epsilon_{0} D_{\mathbf{q}=0}},
$$

allows us to obtain the temperature dependent parameters in $D_{i j}$. We write

$$
\begin{aligned}
D_{\mathbf{q}=0} & =\sum_{j} D_{i j} \\
& \approx D_{0}-D_{1}\left(1-\frac{\sqrt{2 \pi} \alpha_{1}}{a_{0}}\right)-D_{2}\left(1-\frac{\sqrt{2 \pi} \alpha_{2}}{a_{0}}\right)
\end{aligned}
$$

Comparing Eq. 24) and Eq. 22 allows us to determine the $T$-dependence of our model parameters. Following Ref. [1, we make the ansatz that $D_{2}$ is $T$-dependent, while $D_{0}$ and $D_{1}$ are constant.

\section{Numerical Solution of the Model}

In most cases, the model can be solved using a straightforward iterative procedure to obtain self-consistent val- ues for the polarization $P_{i}$ and the electron density $n_{i}$. Given an input potential energy $\phi_{i}$, we calculate the charge density $n_{\alpha i \sigma}$ by diagonalizing $\hat{H}$ and the polarization $P_{i}$ by minimizing $\mathcal{U}$. The charge density and polarization are then used to generate an updated potential energy. The cycle is repeated until the input and output potentials are the same. This cycle is unstable to charge-sloshing, and we therefore use Anderson mixing to stabilize the iterative process. [7]

In some of the cases, the iterative scheme described above is unstable because the flexoelectric contribution to $\tilde{E}$ can lead to a rapid switching of the polarization direction from one iteration to the next. To stabilize the numerics, we rearrange Eq. 21 such that the depolarizing fields are explicitly grouped with $\Lambda_{n}$. Thus, from Eq. 111 it is possible to write $E_{i}=-P_{i} / \epsilon_{\infty}+E_{i}^{\text {other }}$, from which it follows that Eq. 21 is

$$
\left(\Lambda_{n}+\epsilon_{\infty}^{-1}\right) \mathcal{P}_{n}-\mathcal{E}_{n}^{\text {other }}+\gamma \mathcal{P}_{n}^{3}=0
$$

In our calculations, $\Lambda_{n}>-\epsilon_{\infty}^{-1}$ and this rearrangement of terms thus helps stabilize the iterative cycle.

* billatkinson@trentu.ca

[1] G. Khalsa and A. H. MacDonald, Phys. Rev. B 86, 125121 (2012)

[2] S. James Allen, et al., Phys. Rev. B 88, 045114 (2013).

[3] Amany Raslan, et al., Phys. Rev. B 95054106 (2017).

[4] J. Dec, W. Kleemann, and B. Westwanski, J. Phys. Cond. Mat. 11, L379 (1999).

[5] H. Uwe and T. Sakudo, Phys. Rev. B 13, 271 (1976).

[6] A. M. Olés, Phys. Rev. B 28, 327 (1983).

[7] V. Eyert, J. Comp. Phys. 124, 271 (1996). 